International Journal of Modern Physics E

(C) World Scientific Publishing Company

\title{
Jet results and jet reconstruction techniques in $p+p$ and their prospects in heavy-ion collisions in ATLAS
}

\author{
Martin Spousta for the ATLAS Collaboration \\ Institute of Particle and Nuclear Physics, Charles University in Prague, \\ V Holešoviskách 2, 18000 Prague, Czech Republic \\ spousta@ipnp.troja.mff.cuni.cz \\ Received (received date) \\ Revised (revised date)
}

\begin{abstract}
We present the measurement of jet production performed with the ATLAS detector in proton-proton collisions at center-of-mass energy of $7 \mathrm{TeV}$, using an integrated luminosity of $17 \mathrm{nb}^{-1}$. We show the inclusive jet cross sections and jet shapes. The expected performance and strategy for the jet reconstruction in heavy ion collisions is also discussed.
\end{abstract}

\section{Jet production in proton-proton collisions at $7 \mathrm{TeV}$}

Jet rates and cross sections are important tools for understanding the strong interaction. They also provide a measurement of the strong coupling constant and can be used in searches for physics beyond the Standard Model. In this section we will show the first measurement of inclusive single-jet and di-jet cross sections measured in $7 \mathrm{TeV}$ proton-proton collisions with the ATLAS detector. The measurements were performed using data taken between 30 March and 5 June 2010 which correspond to an integrated luminosity of $\approx 17 \mathrm{nb}^{-1} 1$. At the end of this section we will also show the first measurement of the jet shapes using the same data.

For the jet reconstruction, the anti- $k_{\mathrm{T}}$ algorithm was used. It recombines two input objects $i$ and $j$ based on finding the smallest entry in the list of values of variables $d_{i}$ and $d_{i j}$, defined as

$$
d_{i j}=\min \left\{p_{\mathrm{T}, i}^{-2}, p_{\mathrm{T}, j}^{-2}\right\}(\Delta R)_{i j}^{2} / R^{2}, \quad d_{i}=p_{\mathrm{T}, i}^{-2}
$$

where $(\Delta R)_{i j}=\sqrt{\left(y_{i}-y_{j}\right)^{2}+\left(\phi_{i}-\phi_{j}\right)^{2}}$ is the opening angle between object $i$ and $j$. The rapidity $y$ is defined using the energy $E$ and the component of momentum with respect to the beam direction $p_{\mathrm{z}}$, as $y=\frac{1}{2} \ln \left[\left(E+p_{\mathrm{z}}\right) /\left(E-p_{\mathrm{z}}\right)\right]$. If the smallest entry is $d_{i j}$, the input objects $i$ and $j$ are combined and the list is remade. If the smallest entry is $d_{i}$ then the object $i$ is considered to be a final jet. The anti$k_{\mathrm{T}}$ algorithm contains only one free parameter $R$, which sets the relative distance 
at which two jets are resolved from each other. Two values of $R$ are considered, $R=0.4$ and $R=0.6$. The fact that the anti- $k_{\mathrm{T}}$ algorithm recombines input objects by comparing inverse values of their transverse momenta leads to a recombination sequence that starts from the hardest input objects, i.e. from the core of a jet, and ends at the jet periphery, on average at the distance $R$ from the jet axis.

The input objects to the jet finding are clusters of topologically connected calorimeter cells ("Topological Clusters"). Starting from significant signal seeds, the clustering algorithm collects neighboring cells as long as their signals are significant when compared to the noise. The baseline calibration of cells is taken from the test-beam measurements and it corrects the measured energy to the electromagnetic energy scale. Since the ATLAS calorimeters are non-compensating, which means that they have a lower response to hadrons than to electromagnetic energy, the measured jet energy has to be corrected to the hadronic scale. The final jet energy scale calibration and its uncertainty has to account for uncertainties in the modeling of hadronic showers in the detector, the inactive regions in the calorimeter, the description of the calorimeter noise, and uncertainties in the translation of the test-beam energy scale to the in-situ calorimeter (e.g., particles not contributing to the calorimeter signals due to magnetic field bending and dead material, inefficiencies in calorimeter clustering and jet reconstruction) ${ }^{3}$. Several calibration schemes have been developed in ATLAS ${ }^{4}$ :

- Simple $p_{\mathrm{T}}$ and $\eta$-dependent calibration scheme (EM+JES calibration): Jets are found from clusters or towers at the electromagnetic (EM) scale and the calibration constants are applied as a function of the uncalibrated jet $p_{\mathrm{T}}$ and $\eta$. The calibration constants are calculated from Monte Carlo (MC), but they can be derived also using $\gamma+$ jet balance and di-jet balance techniques.

- Global sequential calibration scheme (GS calibration): The jet response is first calibrated with the EM+JES calibration. Then the properties of jets (the energies deposited in different longitudinal segments of calorimeter, the width of the jet) are used to reduce fluctuations in the jet energy measurement without changing the mean value of the response.

- Global cell energy-density weighting calibration scheme (GCW calibration): The cell energy densities are used to calculate the calibration weights. The weights are determined by minimizing the energy fluctuations between the reconstructed and truth jets in MC simulation. Jets are found from clusters or towers at EM scale, then cells are weighted and an additional correction for the dead material is applied. Finally a $p_{\mathrm{T}^{-}}$and $\eta$-dependent correction is added to ensure that the jet energy is properly reconstructed.

- Local cluster weighting calibration scheme (LCW calibration): Clusters are classified according to their topology, then each cell is weighted according to cluster and cell energy density. The weights are determined from MC simulations of charged and neutral pions. Finally additional corrections are applied to take into account energy deposited outside of cluster and the energy deposited in the dead 
material.
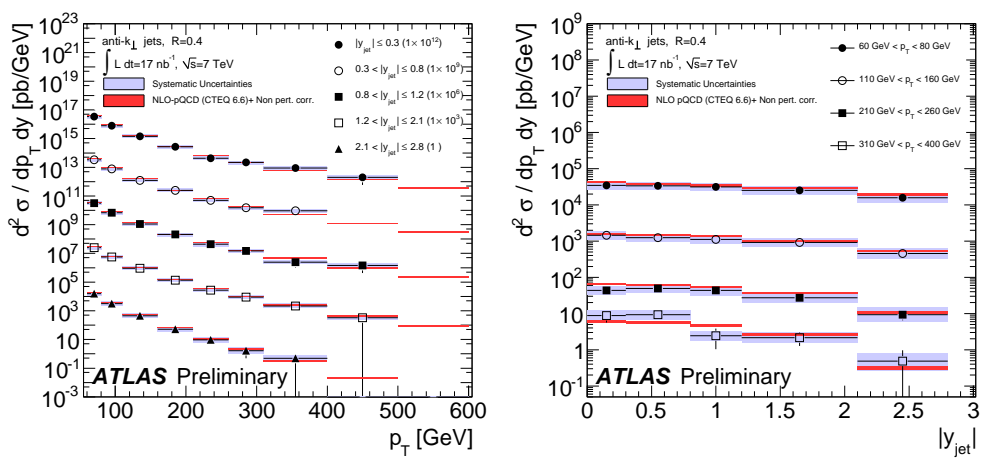

Fig. 1. Inclusive single jet double-differential cross section as a function of $p_{\mathrm{T}}$ for different $|y|$ ranges (left). Inclusive single jet double-differential cross section as a function of $|y|$ for different $p_{\mathrm{T}}$ intervals (right). Data are compared to NLO QCD calculations, corrected for non-perturbative effects.

For this measurement the first calibration scheme has been used. The overall calibration correction on the $p_{\mathrm{T}}$ of jets produced in the barrel region $(0.3<|\eta|<$ 0.8 ) with $p_{\mathrm{T}}>60 \mathrm{GeV}$ is around $50 \%$. With increasing jet energy the size of the correction decreases and for the higest $p_{\mathrm{T}}=500 \mathrm{GeV}$ it is smaller then $30 \%$. The size of the model dependent uncertainty due to hadronization, parton shower, and underlying event has been estimated by comparing the correction derived using a sample of Pythia $6^{5}$ to that derived using a sample of AlpGen + Herwig $6+$ Jimmy 6,7 . It was found to be below $3 \%$. The overall jet energy scale uncertainty is below $9 \%$ over the entire pseudorapidity range, and below $7 \%$ for mid-rapidity jets with $p_{\mathrm{T}}>60 \mathrm{GeV}$.

Among reconstructed jets possible "fake" jets may appear. These fake jets come e.g. from a coherent calorimeter noise, or noise-burst, or the out-of-time energy deposition. These fake jets can be successfully identified and rejected based on a measurement of different qualitative characteristics of jets, here the fraction of energy deposited in different calorimeter layers, minimum number of calorimeter cells containing $90 \%$ of the jet energy, the jet timing, and the quality of calorimeter pulses.

The detector level cross-section has to be corrected to the particle level. This is done in a single step by applying a bin-by-bin correction derived from Pythia $\mathrm{MC}$ simulations. The correction factor which is applied in each bin of a given distribution is defined as $C=\sigma_{\text {truth }} / \sigma_{\text {reconstructed }}$. This procedure is justified by the good modeling of trigger efficiencies in $\mathrm{MC}$ and by the fact that the energy flow around the jets is also well described as we will show at the end of this section. The 
size of the correction varies between $15 \%$ at low $p_{\mathrm{T}}$ and $5 \%$ at high $p_{\mathrm{T}}$. Systematic uncertainties to these corrections were derived by varying the jet energy and position resolution. The uncertainty from the modeling of the cross-section shape which leads to bin-by-bin migrations has been also evaluated. The overall unfolding correction uncertainties were found to be smaller then $5 \%$ in the whole $p_{\mathrm{T}}$ and rapidity range.

The left plot of Fig. 1 shows the inclusive single jet double-differential cross section as a function of $p_{\mathrm{T}}$ for different $|y|$ ranges. The right plot shows the inclusive single jet double-differential cross section as a function of $|y|$ for different $p_{\mathrm{T}}$ intervals ${ }^{1}$. Systematic errors on the measurement are due to luminosity uncertainty $(11 \%)$, uncertainty on the unfolding correction factors $(<5 \%)$, and the jet energy scale uncertainty $(<9 \%)$. Due to the steeply falling $p_{\mathrm{T}}$ spectrum of the inclusive jet distribution the systematic uncertainty is dominated by the jet energy scale uncertainty. The data are compared to NLO perturbative QCD calculations corrected for non-perturbative effects. The uncertainty on theoretical calculations, shown in orange, is the quadratic sum of uncertainties from the choice of renormalization and factorization scales, parton distribution functions, $\alpha_{s}\left(M_{Z}\right)$, and the modeling of soft QCD effects. The cross section extends from $p_{\mathrm{T}}=60 \mathrm{GeV}$ up to around $p_{\mathrm{T}}=500 \mathrm{GeV}$, thus decreasing by more than four orders of magnitude over this range. For both $\mathrm{R}=0.4$ and $\mathrm{R}=0.6$, data and theory are consistent. The di-jet cross section has also been measured. The di-jet mass extends up to $2 \mathrm{TeV}$. The measurement of di-jet cross section is also well described by NLO perturbative QCD calculations corrected for non-perturbative effects.
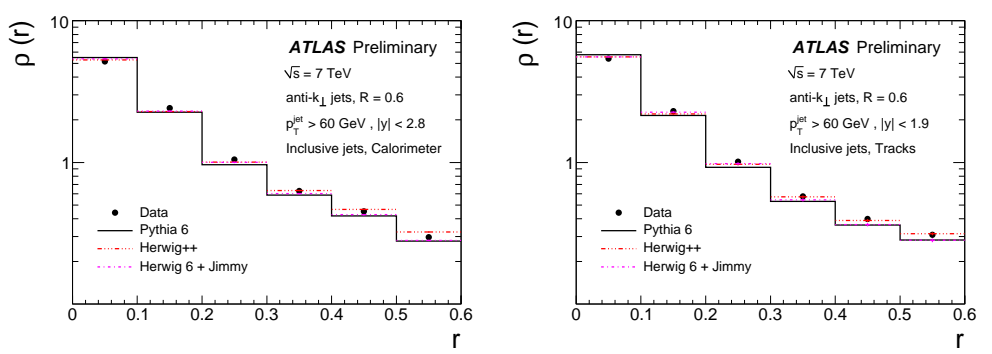

Fig. 2. Uncorrected jet shape reconstructed using the full calorimeter reconstruction (left) and charged tracks only (right) as a function of the radial distance to the jet axis, $r$.

Another task of the precision QCD physics is to measure the internal structure of jets. One basic quantity characterizing the energy and momentum flow within jets is the differential jet shape, defined as the fraction $\rho$ of the jet momentum contained within a ring of thickness $\Delta r=0.1$ at a radius $r=\sqrt{(\Delta \eta)^{2}+(\Delta \phi)^{2}}$ around the jet center, divided by $\Delta r$. The uncorrected jet shapes measured using calorimeter clusters and tracks are shown separately in Fig. 2 for the anti- $k_{\mathrm{T}}$ jets 
with $R=0.6$. The jets simulated by Pythia 6 are slightly narrower than the jets in the data, while the Herwig $6+$ Jimmy and Herwig++ simulations provide a better description. We can conclude that the distribution of the energy within jets is reasonably well described by the simulations.

\section{Jet measurements in heavy ion collisions}

Jet reconstruction in the heavy ion environment differs from proton-proton by a presence of a sizable underlying event. Even if the theoretical predictions on particle multiplicity varies largely, $\mathrm{d} N / \mathrm{d} \eta \in\langle 1000 ; 6000\rangle$, the overall energy deposited in the calorimeter will be significantly higher compared to proton-proton events. This implies that the heavy ion jet reconstruction cannot use default strategies adopted in $\mathrm{p}+\mathrm{p}$ jet reconstruction.

Currently, the two jet reconstruction algorithms are being explored: a seeded cone algorithm and the fast anti- $k_{\mathrm{T}}$ algorithm. The cone and anti- $k_{\mathrm{T}}$ algorithms differ significantly in the way they find jets, in their sensitivity to the underlying event fluctuations, and also in the way the background subtraction can be implemented. For the cone algorithm the background subtraction has to be done before the actual jet finding, for the anti- $k_{\mathrm{T}}$ it is recommended to subtract the background after the jet finding ${ }^{8}$. The anti- $k_{\mathrm{T}}$ algorithm is relatively new and its performance in the large multiplicity environment has not been studied in details yet. Even if the seeded iterative cone is disfavored for precise QCD studies because of its infrared and collinear sensitivity, its application in heavy ion collisions can serve as a basic cross-check of good understanding of the influence of heavy ion underlying event on the jet finding.

The goal of the heavy ion jet reconstruction task is to use algorithms developed for $\mathrm{p}+\mathrm{p}$ measurements so that the basic calibrations studied in $\mathrm{p}+\mathrm{p}$ can be used for $\mathrm{Pb}+\mathrm{Pb}$ measurements with only some modest adjustments. The main requirements on the calibration are the following: they should not depend on the jet substructure which can be modified by the jet quenching, they should provide a possibility to correct the hadronic response before the background subtraction and jet finding, they should provide a basic set of hadronic weights that are not very sensitive to the change in the jet shape and jet size.

The important aspect of the heavy ion collision is that it will not be possible to investigate quickly the effect of the hadronic calibration on the jet energy scale by a comparison between MC and data because there is no good model description of the underlying event. It is very likely that all the available MC programs simulating the soft part of the heavy ion collision will provide a very imprecise description of the real heavy ion interactions. Bearing in mind these facts we decided to use as a primary calibration the global cell weighting characterized above. Since the determination of the jet energy scale uncertainty is non-trivial in heavy ion collisions it is important to study the quantities that exhibit none or only a small dependence on the jet energy scale. 
To subtract the heavy ion underlying event we have tested several techniques. The best performance is provided by the calorimeter segmentation dependent and pseudorapidity dependent background subtraction. This background subtraction can be performed either before the jet finding or after the jet finding. In the former case the fast sliding window algorithm is used to identify regions where jets could possibly reside. These regions are then excluded from the background determination. In the latter case the background determination is "seeded" by fully reconstructed jets. The background is then determined as a function of pseudorapidity in each calorimeter layer separately by summing contributions from cells in $\Delta \eta=0.1$ bins that are outside of the reconstructed jets.

The performance of the heavy ion jet reconstruction has been tested by embedding Pythia di-jet events into Hijing ${ }^{9}$ events. Hijing events were initiated with $\sqrt{s_{N N}}=5.5 \mathrm{TeV}$, without quenching, and with varying centralities. In the most central collisions $(\mathrm{d} N / \mathrm{d} \eta \approx 2700)$, we found the jet energy resolution being better than $20 \%$ for jets above $100 \mathrm{GeV}^{10,11}$. We have also tested the efficiency and rejection of fake jets coming from correlated fluctuations of the underlying event. The efficiency exhibits only a modest sensitivity on the collision centrality. Above jet energy of $90 \mathrm{GeV}$, the efficiency is better than $90 \%{ }^{12}$. Without any fake rejection the fake rate is negligible above $100 \mathrm{GeV}^{13}$. We have also investigated the influence of the underlying event on the ability to reconstruct the jet internal structure. We found that after background subtraction and applying corrections on efficiency and jet position resolution we can well measure the jet internal structure both at the calorimeter level and particle level ${ }^{14}$.

\section{References}

1. ATLAS Collaboration, Measurement of jet production in proton-proton collisions at $7 \mathrm{TeV}$ centre-of-mass energy with the ATLAS Detector, CERN-PH-EP-2010-034, arXiv: 1009.5908

2. M. Cacciari, G. Salam, and G. Soyez, JHEP 0804 (2008) 063.

3. ATLAS Collaboration, Jet energy scale and its systematic uncertainty for jets produced in proton-proton collisions at $\sqrt{s}=7 \mathrm{TeV}$ and measured with the ATLAS detector, ATLAS-CONF-2010-056

4. ATLAS Collaboration, Properties of Jets and Inputs to Jet Reconstruction and Calibration with the ATLAS Detector Using Proton-Proton Collisions at $\sqrt{s}=7 \mathrm{TeV}$, ATLAS-CONF-2010-053

5. T. Sjostrand, S. Mrenna, and P. Skands, JHEP 05 (2006) 026.

6. G. Corcella et al., JHEP 01 (2001) 010.

7. J. M. Butterworth, J. R. Forshaw, and M. H. Seymour, Z. Phys. C72 (1996) 637646.

8. M. Cacciari (2006), hep-ph/0607071

9. M. Gyulassy and X.-N. Wang, Comput. Phys. Commun. 83 (1984) 307

10. M. Spousta for the ATLAS Collaboration, ATL-PHYS-PROC-2010-069.

11. N. Grau for the ATLAS Collaboration, J. Phys. G 35 (2008) 104040.

12. M. Spousta for the ATLAS Collaboration, ATL-PHYS-PROC-2009-046.

13. N. Grau et al., arXiv:0810.1219.

14. M. Spousta for the ATLAS Collaboration, ATL-PHYS-PROC-2009-002. 